\title{
ESTRATEGIAS PARA LA TRANSFORMACIÓN DEL DOLOR DESDE LA EXPERIENCIA DE MUJERES AFROCOLOMBIANAS VÍCTIMAS SOBREVIVIENTES
}

Strategies for the Transformation of Pain from the Experience of Afro-Colombian Women Survivors

\author{
Alba lucía Cruz Castillo, Alejandra Calderón, Isabela Castellano, Sonia Flórez
}

Universidad de La Salle, Colombia

KEY WORDS

Sexual Violence

Intersectionality

Pain

Agency

\section{ABSTRACT}

This article gives an account of the processes undertaken by AfroColombian women who are survivors of sexual violence and displacement in the context of the Colombian conflict to heal their pain, in which, using their ancestral knowledge, their ways of weaving community and relating, they empower political processes, of visibility and resistance to a violence that has not only penetrated the body but also its family and community nucleus. The article therefore focuses on the processes that women have developed from their role as surviving victims and from which it is possible to reflect on the different intersections present when reading violence towards them.
PALABRAS CLAVE

Violencia sexual Interseccionalidad Dolor

Agenciamiento

\section{RESUMEN}

El presente artículo da cuenta de los procesos agenciados por mujeres afrocolombianas víctimas sobrevivientes de violencia sexual $y$ desplazamiento en el contexto del conflicto colombiano para sanar su dolor, en los cuales, haciendo uso de sus saberes ancestrales, de sus formas de tejer comunidad y relacionarse potencian procesos políticos, de visibilización y de resistencia a una violencia que no solo ha traspasado el cuerpo sino también su núcleo familiar y comunitario; el artículo por lo tanto se centra en los procesos que desde su rol de víctimas sobrevivientes las mujeres han elaborado y desde las cuales esposible reflexionar sobre las distintas interseccionalidades presentes al leer la violencia hacia ellas. 


\section{A manera de introducción}

$\mathrm{E}$ presente artículo se desarrolla en tres apartados: en el primero se hace un reconocimiento a las huellas históricas que han marcado la violencia hacia la población negra en el país, haciendo mayor hincapié en el caso de las mujeres negras y las múltiples dimensiones de la lectura de esta violencia, sobre todo en el contexto de la guerra; el segundo apartado ilustra los distintos procesos de agenciamiento que han liderado las mujeres víctimas desde su rol de sobrevivientes, en donde han asumido un proceso protagónico en la consolidación de estrategias de sanación al dolor y el último apartado enfatiza sobre los aprendizajes derivados de este proceso que pueden ser tenidos en cuenta para pensarse la reparación desde una perspectiva afroreparadora en el marco de la transición política en Colombia. En este sentido el texto pretende dar algunos elementos para el debate acerca de la necesidad de plantear con mayor énfasis estrategias que le posibiliten a las comunidades negras en Colombia un proceso de reparación real a su dolor y los múltiples daños causados por la guerra.

\section{Huellas del conflicto armado en mujeres afrocolombianas una lectura intersecciones}

\begin{abstract}
¡Pacífico yo! ¿Por qué no soy violento? Pobre de mí, si en mi cabeza no existe armamento. ¡Mi cuerpo y mis pies bailan, un canto alegre, que no tiene sentimiento! (Vallejo, 2015)
\end{abstract}

Los pueblos negros sujetos y sujetas sociales, se han movido en dinámicas de desarraigo relacionadas históricamente con dinámicas de conflicto, en América Latina más específicamente en Colombia una relación directamente con el conflicto armado; en el que la relación de desarraigo se conecta con desplazamiento forzado "El desplazamiento es un dato recurrente y cuasipermanente de la historia colombiana; hace parte de la memoria de las familias y de las poblaciones; está inscrito en los recuerdos de los habitantes urbanos, precedió la fundación de barrios en las grandes ciudades y de poblaciones grandes y pequeñas a lo largo y ancho de las fronteras internas." (Giraldo, 2001, p. 3) el cual conceptualmente Nora Segura Escobar y Donny Meertens lo define como:

El desplazamiento forzado se asocia con diferencias sociales construidas en torno a la nacionalidad, etnoraciales y de clase. Apenas recientemente se ha tomado conciencia sobre otros marcadores sociales de diferencia, como el género y la edad, que suponen formas peculiares de exposición a la violencia, consecuencias y posibilidades diversas en relación con la reconstrucción de proyectos de vida individuales y colectivos. (Segura \& Meertens, 1997, p. 32)

De acuerdo a lo anterior las condiciones etnoraciales se convierten en un foco de selección de víctimas de desplazamiento forzado, el desarraigo se genera con relación a prácticas culturales y étnico ancestrales, en el caso de lo étnico racial se ven afectadas "El desarraigo de ese mundo ha significado destrucción de la identidad social" (Segura \& Meertens, 1997, p. 38) el desarraigo afecta y fractura el tejido social en las diversas dimensiones del sujeto $\mathrm{y}$ de los colectivos, afectando no solo lo social si no también lo emocional que pasa por, una pérdida de identidad que se puede dar en el individuo o en la colectividad afectando la construcción de sujetos políticos; afectaciones que se dan en contexto que implican llegar de una región a otra o en algunos casos más marcados por la transformación de pasar del casco rural al urbano.

El desarraigo también ha sido valorado como un fenómeno de la modernidad capitalista, desde la cual se retoma la fenomenología para una comprensión más allá de, la indiferencia relacionada con los lugares, retomando a Eduard Casey citado por Arturo escobar, "vivir significa vivir en un lugar" (Escobar, 2004, p. 65) , el cual desde una perspectiva antropológica implica desatar la implantación local haciendo referencia a lo territorial de prácticas culturales, a pesar de que el territorio sea un resultado de prácticas históricas que para el caso de ser negro del pacifico implica una conexión directa con la multiculturalidad y lo pluriétnico desde el territorio, estas condiciones y tradiciones culturales que se desarrollan en los territorios construyen a los sujetos y colectivos desde diferentes dimensiones que llegan hasta una 
súper estructura, pero esas construcciones subjetivas se desligan del territorio y pueden ser reconstruidas y replicadas en otros territorios.

Esta dinámica ha marcado a hombres $\mathrm{y}$ mujeres negras a lo largo de la historia, , en la que en más de medio siglo la guerra en Colombia ha dejado muertes, destrucción y profundos dolores humanos, en los que para el 9 de agosto del año en curso las victimas ascienden a 8,7 millones según el registro único de víctimas, pero en el conflicto armado se encuentran múltiples manifestaciones de la violencia en las que a partir de las cifras expuestas por el informe BASTA YA “ entre 1958 y el año 2012 murieron 220.000 personas como consecuencia del conflicto armado(..), esta cifra también permite confirmar que cada tres muertes violentas del país las produce la guerra y durante cinco décadas, en promedio murieron once personas por esta causa." (Centro Nacional de Memoria Historica , 2014, p. 21). En las que las mujeres han sido víctimas destacadas pues entre las múltiples violencias de las cuales han sido víctimas se encuentra la violencia sexual.

La violencia sexual en el contexto de la guerra se lleva a cabo como ejercicio de poder sobre la vida de las mujeres y adicionalmente como; "una manera de recordar que su cuerpo es parte del botín" (Dador, 2007)por lo tanto, los hechos van dirigidos para afectar la vida de la mujer, su cuerpo e integridad personal, donde el control y la acción tiene la finalidad de generar temor y la sensación de obediencia a las normas establecidas por los actores armados.

La violencia sexual a mujeres en el marco del conflicto armado en el caso colombiano se convierta en:

una práctica generalizada en el conflicto armado colombiano, usada de manera sistemática por todas las partes: guerrilleros, paramilitares y miembros del Ejército y de la Policía Nacional; que la utilizan como estrategia de guerra, como forma de tortura o de castigo combinada con prácticas de mutilación contra mujeres acusadas de simpatizar con el enemigo, como mecanismo para humillar al enemigo o junto con modalidades de esclavitud. (p. 12)

La violencia sexual hacia las mujeres hay cobra una multidimensionalidad en la cual, según los testimonios se constituye en una "agresión, un atentado contra la integridad y la autonomía física de las mujeres, representa la apropiación violenta y directa de los cuerpos y de su sexualidad" (Ruta Pacífica de las Mujeres, 2013, p. 23) lo que genera una estrecha relación con la finalidad de generar daños morales, físicos y de fragmentación al proyecto de vida de las mujeres.

Según el informe la verdad de las mujeres víctimas del conflicto armado, "las mujeres más golpeadas por la violencia sexual fueron las afrodescendientes y las mestizas" (Ruta Pacífica de las Mujeres, 2013, pág. 67) y las dinámicas en las cuales se generaba el fenómeno eran de control y establecimiento de un orden social y económico por parte de los grupos armados, en el cual se evidenciaba la militarización de la vida, esclavitud doméstica, acoso sexual y la privación de la participación de las mujeres en actividades de su comunidad.

En el caso de la violencia sexual hacia mujeres negras, es importante el reconocimiento de las dinámicas históricas de discriminación de la étnica y de género, dado que, intervienen procesos de dominación desde la colonización en el siglo XVIII y XIX, en los que se impone el concepto de raza, el cual genera una jerarquía marcada por la subordinación y el establecimiento de normas y estructuras sociales desde Europa, hacia América Latina y en el caso específico de Colombia, tal y como se ha desarrollando al inicio del apartado, pues dichas estructuras sociales de dominación se relacionan con la violencia hacia la mujer, puesto que, a esta se le impone un deber ser a la mujer negra a partir del rol de trabajo doméstico y reproductivo y al hombre varonil - fuerte- que toma decisiones en el ámbito público. (Marciales Montenegro, 2013)

Expresión que se adhiere a la radicalización del sexo, en la que interviene el racismo cultural, estructural, institucional y social. Para la autora la violencia hacia las mujeres negras afrocolombianas en el marco del conflicto armado en Colombia se puede identificar como estrategia militar a través de la cual los grupos armados legales e ilegales alcanzan los objetivos de control, así mismo, como:

(...) instrumento a través del cual se expresa simbólicamente el dominio masculino y racista sobre cuerpos, territorios y poblaciones, razón 
por la cual además de considerarse como una expresión de la violencia de género, debe ser analizada como una clara manifestación del racismo estructural, el cual juega un papel determinante en la configuración y desarrollo del conflicto armado, en el actuar de los actores armados y en el impacto diferenciado que puede ocasionar sobre la población negra, particularmente sobre las mujeres(...)la interacción de las categorías, sexo/genero/ raza debe ser vista como el tejido que en conjunto configura el fenómeno.(Montenegro Marciales , 2015, pág. 86)

En el marco del conflicto armado colombiano se pueden evidenciar las categorías mencionadas por la autora y la reproducción del racismo estructural que viene desde la construcción colonial de la discriminación, exclusión y privación de oportunidades laborales a la población negra afrocolombiana, sumado a la violencia como consecuencia de las actividades de explotación de recursos por el control económico a nivel rural y regional.

La persecución desde los grupos armados a las mujeres afrocolombianas por sus prácticas de la ancestralidad se suma a la violencia psicológica y sexual que trajo la militarización de la vida de las mujeres ocasionando transformaciones para evitar hechos de violencia hacia su integridad, la de sus familias $y$ comunidad. Por último, es importante señalar algo en especial y es que la violencia sexual hacia las mujeres negras afrocolombianas también se llevó a cabo como amenaza a la defensa de derechos caso en el cual "los perfiles de liderazgo fueron perseguidos". (Montenegro Marciales, 2015, pág. 84).

Las mujeres negras han sido objeto de violencia de múltiples maneras: a manera de desplazamiento, de desarraigo y de violencia sexual; daños que en ocasiones han sido reconocidos por el Estado y otras ocasiones no, razón por la cual se han visto en la necesidad de agruparse, hablar de su dolor y a la vez buscar alternativas desde donde sanarlo, este es el caso de AFROMUPAZ (Asociación de mujeres afro por la paz) organización declarada bajo la ley 1448 como sujeto de reparación colectiva y que desde el uso de saberes ancestrales agencia procesos para transformar el dolor.

\section{Procesos de Agenciamiento Político para la Transformación del dolor}

Pero como el ave fénix me volveré a levantar, uniré mi voz con otras, donde los derechos humanos de las mujeres sean su prioridad. (Afromupaz, 2014, p. 33)

Para hacer frente a las experiencias de sufrimiento por las que han pasado las mujeres negras afrocolombianas, son ellas mismas quienes han liderado sus procesos para transformar el dolor en oportunidades de sanación y reparación de sus heridas. Estos mismos procesos de carácter colectivo, social y político surgen bajo diferentes factores tales como las barreras y las rupturas que se presentan en el acompañamiento del Estado hacia las víctimas del conflicto armado en Colombia y a la percepción de rol pasivo que les han otorgado generando que sean las mismas mujeres quienes agencien iniciativas y acciones para la reivindicación de sus derechos y su reconocimiento como sobrevivientes.

Ahora bien, bajo un enfoque diferencial étnico, que se ajusta a las particularidades de los sujetos y reconoce los saberes ancestrales y la cosmovisión de las comunidades negras (CODHES, 2017, p. 17), Afromupaz orienta iniciativas, procesos colectivos $y$ acciones políticas para la construcción y el posicionamiento en una plataforma que permita visibilizar con un rol activo frente a su reparación colectiva desde su sentido de pertenencia con el territorio de origen y su identidad como víctimas-sobrevivientes.

El agenciamiento político se comprende como una construcción teórica que Gidenns citado por López (2004, p. 17) define como la capacidad reflexiva para actuar, de tal manera, que agenciar implica una conciencia práctica en la que se racionaliza y moviliza paralelamente no solo desde un individuo sino también desde un colectivo que identifica sus derechos y la vulneración de estos. Asimismo, las mujeres negras víctimas sobrevivientes integrantes de Afromupaz se reconocen como sujetos políticos, interlocutoras con una voz que reclama sus derechos y exige su protección para luego así lograr transformar sus realidades mediante un análisis crítico y un sentido político. 
Las alternativas para sanar el dolor que las mujeres de Afromupaz han creado para su reparación colectiva comprende de cuatro iniciativas con su respectivo proceso. La primera de estas iniciativas tiene que ver con la reterritorialización, es decir, la resignificación del territorio en el que se construyen espacios nuevos con base en las prácticas del territorio de origen, lo que implica estructurar un territorio no dependiente, más bien, como señala Nussbaum (1995) citado por Zuluaga Tapia (2014, pág 137), un espacio para gobernarse. Afromupaz pasa por el sentir nuevos miedos que emergen de reubicarse en la ciudad y las inquietudes de la posible exclusión (págs. 138139) por lo que reconocer su identidad colectiva se convierte en una forma de reivindicar sus derechos y, al mismo tiempo, de afrontar el rechazo.

La segunda iniciativa, tiene que ver con el uso del patrimonio cultural inmaterial, lo cual implica el preservar y conservar todo aquello intangible que está presente en la cultura y, asimismo, se recrea colectivamente (Ureña Alvarez, 2004, pág. 251), como las tradiciones orales, expresiones artísticas, bailes, y cantos como los alabaos, originados por sus ancestros africanos, que narran experiencias de la cotidianidad entre las comunidades negras afro. El uso de los saberes ancestrales es, entonces, una forma de narrar la memoria, denunciar las violencias estructurales que han vivido históricamente las comunidades negras afrocolombianas y su resistencia, además de hacer visible la acción política conjunta de Afromupaz como colectivo que rechaza las dinámicas de la guerra y sus efectos y aporta a la construcción de paz desde su contexto. Por otro lado, al reconocer los perfiles de las ancestras estos se convierten en una voz fundamental del proceso de sanación, participación y fortalecimiento de su identidad colectiva.

La tercera iniciativa pasa por el autoreconocimiento y la autodeterminación que las mujeres de la Asociación adquieren desde un empoderamiento pacifista y así logran identificar sus fortalezas a la vez que sus derechos. Esto implica la acción noviolenta como factor esencial la cual contribuye con la resolución y el afrontamiento de problemas en relación a diferentes tipos de violencias con la finalidad de movilizar mediante la generación de conciencia y acción crítica desde la comprensión y el reconocimiento de sus capacidades para luego así "negociar, intervenir y participar en mejorar las condiciones en las decisiones que afectan sus vidas y a la comunidad..." (Sandoval Forero, 2015, pp. 87-89), de esta manera, las mujeres y la comunidad agencian control sobre sí mismas y aseguran el respeto por sus derechos además de transmitirlo a las demás generaciones e incidir políticamente.

La cuarta iniciativa tiene que ver con la acción narrativa autobiográfica en la que las mujeres interpelan el dolor mediante relatar sus experiencias de vida en contexto de guerra evidenciando el agenciamiento político al reconocer la importancia sus voces llevando un mensaje de resistencia y vida el cual la memoria tiene un papel para la resignificación del dolor y su rol movilizador y reparador que permita romper el silencio para ir a la acción.

Es importante reconocer, en los procesos de sanación que lleva a cabo Afromupaz, el papel simbólico que adquiere la memoria histórica de carácter colectiva y corporal, convirtiéndose en una estrategia para afrontar el dolor y, a la vez, posicionarse políticamente como mujeres sobrevivientes del conflicto armado colombiano a través de construir en pro de los vínculos, preservar y tejer la vida en colectivo.

Cabe resaltar que este posicionamiento como víctimas-sobrevivientes y el agenciamiento político son nociones estrechamente relacionadas, referentes como Haraway afirma que el agenciar se caracteriza por el compromiso ético-político con una comunidad y un territorio, compromiso que Bohorquez Farfán y Rojas Ariza (2017) reconocen desde la víctima como generadora de cambio social, quién asume un rol activo para la transformación de sí misma/o con incidencia de carácter colectivo; por lo tanto, es desde esta lógica que Afromupaz ha desarrollado acciones a favor de las víctimas contribuyendo a $\mathrm{su}$ reconocimiento en su territorio y el posicionamiento como mujeres afrocolombianas sobrevivientes del conflicto.

La incidencia en los espacios locales de participación hace parte de las acciones que Afromupaz lidera contribuyendo a la construcción de territorialidades de paz, como 
escenarios políticos, que no solo aportan la reconstrucción del proyecto de vida de las mujeres integrantes de la Asociación si no que logran llegar a otros espacios en los que comunidades más amplias sienten la seguridad y tranquilidad.

Por otro lado, Afromupaz cuenta con incidencia nivel distrital ya que realizan el Congreso Cuerpo y Cara de Mujer un espacio que la Asociación desarrolla en alianza con otras entidades entre las que se encuentra Unidad de víctimas, la Agencia de los Estados Unidos para el Desarrollo Internacional (USAID), la Organización Internacional para las Migraciones (OIM), ONU Mujeres, Consejería Presidencial para la Equidad de la Mujer, entre otras. Este congreso busca contribuir a la reconciliación mediante diferentes acciones políticas para reivindicar, exigir y defender sus derechos como víctimas sobrevivientes.

Ahora bien, Afromupaz se identifica como un colectivo de mujeres resistentes, empoderadas y con agencia que han logrado por medio del autocuidado, la solidaridad y los procesos reflexivos individuales y colectivos de reconstrucción memoria "la memoria guardada en el cuerpo se despliega y se re-arma en la escena" (Ruta pacifica de las Mujeres, 2013, pág. 47) y les permite reconocerse como lo hacen hoy en día.

Las iniciativas, procesos y acciones que lidera Afromupaz generan espacios de encuentros seguros en los que principios éticos como la escucha activa y la empatía son pilares fundamentales para sus relaciones y la reconstrucción del tejido social ya que se convierten en elementos que permiten propiciar el apoyo social como estrategia de afrontamiento al dolor, ejercicio de ciudadanías cuidadoras y recuperación lazos de solidaridad y confianza.

\section{Aprendizajes desde la perspectiva de las mujeres víctimas -sobrevivientes para la reparación con enfoque étnico. A manera de conclusión}

Como sobrevivientes de la violencia, consideramos que tenemos un papel muy importante ya que no solo hemos venido trabajando por superar el dolor y reconstruir nuestras vidas, sino que desde nuestras luchas y sufrimientos hemos venido construyendo un mejor país. (Afromupaz, 2014, pág. 31)

El presente apartado busca acercarse a los aprendizajes desde la perspectiva de las mujeres víctimas- sobrevivientes para la reparación con enfoque étnico especialmente desde la experiencia de la Asociación de Mujeres Afro por la Paz, (Afromupaz). Para esto, es preciso hacer referencia a la noción de la etno- reparación y afro reparación; la primera se relaciona con los derechos de los grupos étnicos entre los que se encuentran los afrodescendientes y afrocolombianos y se centra en reparar las acciones que ocasionaron vulneraciones de derechos y por lo tanto se necesita de restaurar las afectaciones de los hechos de los que ha sido víctima el grupo étnico, teniendo en cuenta en el proceso la cosmovisión de los grupos étnicos, intereses y demandas desde la visión de la realidad a partir de su vida cotidiana, cultura y el reconocimiento de impactos particulares. (Rodríguez Garavito \& Lam, 2011).

Según Ogleetree (2003), citado por Garavito y Lam (2011, p. 22) existen dos aspectos centrales en la etno-reparación los cuales son: La rendición de cuentas por los daños ocasionados a las comunidades que en el presente no han sido reparados y el compromiso que tiene la sociedad de proponer medidas que contribuyan a resarcir los daños que no fueron reparados, Acá inicia el rol de la afro reparación ya que se articula desde la resistencia histórica de las comunidades afro frente a la discriminación racial, exclusión y las afectaciones mayoritarias a las comunidades afrocolombianas en el marco del conflicto armado en Colombia.

En relación con lo anterior la Afro-reparación se convierte en una necesidad que busca reconocer los daños históricos ocasionados a las comunidades afrodescendientes, así como a sus territorios objeto directo de violación de Derechos Humanos, "en especial a los derechos a la libertad, a la vida y a la integridad personal" (Miranda, 2007, p. 426), sumado al control económico e intereses políticos dominantes. Es así como la afro reparación dese las mismas comunidades afrocolombianas se ubica en las acciones que crean para la supervivencia $y$ reconocen desde sus saberes, experiencias y 
cosmovisión un marco que guie sus propuestas del cumplimiento de la verdad, la justicia, reparación y no repetición con el fin de esclarecer los hechos y plantear estrategias que logren resarcir el sufrimiento ocasionado por el conflicto armado.

La experiencia de Afromupaz, deja aprendizajes en los cuales se hace visible las demandas permanentes de la asociación frente a la vulneración de derechos y la continuidad de hechos violentos que buscan amenazar su capacidad organizativa, la integridad de sus lideresas y líderes y por otro lado frustrar el proceso colectivo, también, a partir de la creación de acciones para la supervivencia y reparación una de las característica principales de la afro-reparación se tiene en cuanta en la experiencia de Afromupaz dado que, desde sus saberes, la resignificación del territorio, el uso del patrimonio cultural inmaterial, y la experiencia narrativa biográfica que se da en la "Huerta al Perejil" estrategia de acompañamiento psicosocial afrocolombiano a las heridas ocasionadas por el conflicto armado en Colombia, se permite aportar significativamente a la etno- reparación y hacer realidad en la cotidianidad acciones conjuntas que se ubican en la afro- reparación.

Transversal a las estrategias de agenciamiento político y las alternativas en torno a la reparación que han creado en Afromupaz se tiene presente la identidad cultural que es un elemento importante para la reparación de cada grupo étnico, así mismo el reconocimiento de la naturaleza colectiva de los hechos victimizante, sus consecuencias y las necesidades particulares como mujeres, en sus familias y el grupo que integra dicha asociación, aspecto que también es esencial como aprendizaje para la reparación en otros colectivos afrocolombianos.

Se puede identificar como las tradiciones culturales y orales han sido una herramienta esencial en el proceso de sanación y reconstrucción de memoria colectiva e histórica, puesto que, se han convertido en repertorios simbólicos de la memoria que a través del canto popular y los alabaos que hacen parte del patrimonio cultural inmaterial se aporta a la preservación de las tradiciones orales, creación de acciones de resistencia a la guerra y la preservación de la identidad colectiva donde las propuestas alternativas de reparación implementadas por Afromupaz aportan al acompañamiento a las vulneraciones de derechos frente a las afectaciones de carácter colectivo como los son la fractura de las formas de solidaridad de las comunidades y la sostenibilidad del patrimonio cultural.

De otro lado, la recuperación del territorio ancestral y la identificación de la reterritorialización como una apuesta política y organizativa de las comunidades afrocolombianas que se han visto enfrentadas a las dinámicas del despojo. Es esencial Pensarse a modo de aprendizaje del cual suscita un desafío; el acompañamiento a nivel comunitario desde el respeto de la forma de ser, de crear y recrear la cultura de cada población y grupo étnico, propiciando espacios de encuentros seguros y entretejiendo otras formas de acompañar el dolor, de identificar y movilizar redes de apoyo social desde procesos en los cuales el trabajo social en su rol de mediador y agente de cambio que se articula con la dimensión socioeducativa, ético política, así como sociohistórica donde se moviliza en torno a la búsqueda de nuevas realidades del quehacer técnico y la importancia del acercamiento a las comunidades como aspecto fundamental para la lectura siempre crítica y multidimensional de los contextos y las cosmovisión de grupos étnicos especialmente los que nos convocaron a llevar a cabo la investigación Afrocolombianos.

Por último, la investigación convoca en la academia y el quehacer profesional a profundizar en la afro-reparación durante el proceso de investigación lograr un acercamiento a su práctica en la cotidianidad desde las comunidades y su visión desde su experiencia la cual nos lleva a reconocer que el acercarse a la afro- reparación como desafío en trabajo social requiere de una mirada crítica del desarraigo histórico de comunidades afrodescendientes en el mundo y en Colombia y la dignidad como derecho de las comunidades para la preservación de las tradiciones culturales y centralidad en los procesos de reparación. A si como, el ejercicio de la praxis del cuidar en la cotidianidad en el caso de Afromupaz se relaciona con la politización del dolor que ha permitido a las mujeres romper el 
silencio y compartir su experiencia de vida con otras mujeres para que se generen procesos de interpelación del dolor a través de la experiencia narrativa y de esta manera aportar a la denuncia, empoderamiento pacifista y agenciamiento político como la capacidad reflexiva para actuar pensando en la no repetición de hechos violentos, en el acompañamiento al dolor a través de la recuperación de lazos relacionales y de confianza, y a la movilización frente a la construcción de estrategias alternativas para la reparación, exigencia de derechos del colectivo y mejoramiento de su calidad de vida. 
Estrategias para la transformación del dolor desde la experiencia de mujeres afrocolombianas...

\section{Referencias}

Afromupaz. (2014). Verdades ancestrales: La huerta al perejil. Bogotá, Colombia: Defensoria del Pueblo. Afromupaz. (2014). La Huerta al Perejil. Bogotá: Defensoría del Pueblo.

Bohórquez Farfán, L., \& Rojas Ariza , Y. H. (2017). De víctimas a sobrevivientes: el reto de la reconstrucción de memoria histórica en Colombia. Revista Cambios y Permanencia , 717-735.

Centro Nacional de Memoria Historica . (2014). ¡BASTA YA! Colombia memorias de guerra y dignidad resumen. Bogota : CNMH.

CODHES. (2017). Ajustes urgentes a la ruta étnica de reparación colectiva. In CODHES, REPARACIÓN COLECTIVA Y PAZ TERRITORIAL: Una propuesta para ajustar la actual ruta administrativa en el marco del acuerdo entre el gobierno y las Farc -EP (pp. 17-21). Bogotá: CODHES.

Corporación Humanas, Centro Regional de Justicia de Género . (2009). Situacion en Colombia de la violencia contra las mujeres. Bogotá, Colombia : Ediciones ÁNTROPOS .

Ema López, J. E. (2004). Del Sujeto a la Agencia (a través de lo político). Athenea Digital , 1-24.

Escobar, A. (2004). Desplazamientos, desarrollo y modernidad en el Pacífico colombiano. In E. Restrepo, \& A. Rojas , Conflicto e (in) vicivilidad retos en los estudios de la gente negra en colombia (pp. 53-72). Popayan: Universidad del cauca.

Dador, J. (2007). Impunidad pongámosle fin: Violencia Sexual contra las mujeres en el conflicto armado y post conflicto en Ámerica Latina. (C. e. Proyectos, Ed.) Retrieved Febrero 18, 2018, from acnur.org: www.acnur.org/filedmin/scripts/doc.php?file=fileadmin/Documentos/Publicaciones/2007/4739 \#_ga2.19145596.516926357.1519086051-174840939.1519086051

Giraldo, G. N. ( 2001). El desplazamiento forzado en Colombia. Reinvención de la identidad e implicaciones en las culturas locales y nacional. Revista Electrónica de Geografía y Ciencias Sociales, 1-17.

Marciales Montenegro, C. X. (2013). Violencia Sexual en el Conflicto Armado: Los rostros afro de la reparación. Caso: Asociación de Mujeres Afro por la Paz. Bogotá: Universidad Nacional de Colombia.

Mbembe, A. (2016). Critica de la Razon Negra. España: Ned Ediciones.

Miranda, L. G. (2007). Desde adentro: una aproximación al tema de Verdad, Justicia y Reparación a partir de las víctimas afrocolombianas. In C. M. Rosero-Labbé, \& L. C. Barcelos, Afroreparaciones:Memorias de la Esclavitud y Justicia Reparativa para negros, afrocolombianos $y$ raizales (pp. 423 - 441). Bogotá: Colecciones CES.

Ruta Pacífica de las Mujeres. (2013). La verdad de las mujeres víctimas del conflicto armado . Bogotá: Comisión de Verdad y Memoria de Mujeres colombianas.

Rodríguez Garavito, C. \& Lam, Y. (2011). ETNOREPARACIONES: La justicia colectiva étnica y la reparación a pueblos indigenas y comunidades afrodescendientes en Colombia. Bogotá: DeJuSticia.

Sandoval Forero, E. A. (2015). Empoderamiento pacifista para otros mundos posibles. Revista de Paz y Conflictos, 75-95.

Segura, N. E. \& Meertens, D. ( 1997). Desarraigo, género y desplazamiento interno en Colombia. Nueva Sociedad, 30-43.

Ureña Alvarez, R. (2004). La protección del patrimonio cultural en tiempo de guerra y de paz. Revistas Cientificas Complutenses (14).

Vallejo, C. (2015). Mil poemas. Chile: Alfred Asís. 